\title{
Memórias sobre tempos de peste: a linguagem Guaraní do século XVII das doenças e epidemias (parte 1)
}

\author{
Plague time memories: 17 th century Guaraní language on \\ diseases and epidemics (part 1) \\ Francisco Silva Noelli ${ }^{1}$ \\ https://orcid.org/0000-0003-0267-583X
}

DOI: $10.26512 /$ rbla.v12i1.35064

Recebido em setembro/2020 e aceito em outubro/2020

\begin{abstract}
Resumo
O registro linguístico sobre saúde e doença nas línguas Guaraní começou no século XVII. Este artigo reúne 308 palavras e frases levantadas nos dicionários de Antonio Ruiz de Montoya, publicados nos anos 1639 e 1640. Além do interesse direto para medicina e saúde pública, também é fonte para a linguística histórico-comparativa e para a história da medicina Guaraní praticada milenarmente por kuña paje e pajes, mulheres e homens sábios na arte de curar.
\end{abstract}

Palavras-chave: Brasil Meridional. Língua Guaraní. Medicina Guaraní. Saúde Guaraní.

\begin{abstract}
The written linguistic record on health and disease in Guaraní languages began in the 17th century. This article assembles 308 words and phrases collected in the dictionaries of Antonio Ruiz de Montoya, published in the years 1639 and 1640. In addition to the direct interest of medicine and public health, it is also a source for historical-comparative linguistics and for the long-term history of the Guaraní medicine practiced by kuña paje and paje, the wise women and men in the art of healing.
\end{abstract}

Keywords: Southern Brazil. Guaraní language. Guaraní medicine. Guaraní health.

"Não há língua bárbara, senão para quem não a entende."

Bartomeu Melià, 1995

Este artigo mostra alguns registros sobre doença na língua Guaraní da antiga região do Guairá, feitos por um missionário da primeira metade do século

1 Doutorando em Arqueologia, Universidade de Lisboa. E-mail: francisconoelli@edu. ulisboa.pt. 
XVII. Ele testemunhou ao longo da vida mortandades e sofrimento humano causados por epidemias, sendo acometido por tifo e, ao menos uma vez, pela malária (Jarque 1900). Portanto, teve experiências e sentimentos semelhantes aos dos viventes deste ano de 2020, um verdadeiro tempo de peste, onde toda a humanidade foi afetada pela pandemia de COVID-19, ou corona virus disease 19, nome dado pela da Organização Mundial da Saúde para o vírus SARSCoV-2 (Coronavírus da Síndrome Respiratória Aguda Grave 2), passando a conhecer melhor o que é o contágio, contaminação e as suas consequências físicas e emocionais, deixando mais compreensível o esforço linguístico de Antonio Ruiz de Montoya. Ele registrou minuciosamente um extenso repertório sobre o tema nos dicionários Tesoro e Vocabulario de la lengua Guaraní, publicados em 1639 e 1640, permitindo acesso direto ao vocabulário cotidiano daquela época. É útil agora para a história, revelando um filtro e seleção de informações a serviço do colonialismo, como um guia para outros missionários se comunicarem objetivamente em campo. Também é útil às memórias Guaraní, para as pessoas do presente considerarem o que diziam seus antepassados, tal como registrou o linguista plenamente competente. Montoya reuniu detalhes únicos, não descritos por outras fontes, como revela a portentosa introdução feita por Bartomeu Melià (2011) sobre a composição dos dicionários.

A linguagem que se mostra vai para além dos interesses Guaraní e da investigação linguística, histórica e antropológica. Ela possui utilidade médica agora, especialmente na intermedicalidade, valendo igualmente ao objetivo do Vocabulário Guarani-Español para uso médico de Gatti, Rojas e Bertoni (1947:5), composto "para recoger y apreciar los sintomas subjetivos y muchas circunstancias de la salud y de las enfermedades, el médico debe saber interrogar en guarani y, sobre todo, saber interpretar las manifestaciones de los enfermos". Esses autores, na primeira metade do século XX, mostraram a vitalidade da linguagem registrada por Montoya. E, um deles (Gatti 1985), após décadas de prática médica atendendo guaranis de todo o Paraguai, concluiu em 1955 as 10 mil entradas da Enciclopédia Guarani-Castellano de Ciencias Naturales y Conocimientos Paraguayos, incluindo os registros do missionário, tanto os que ainda estavam em uso, quanto os considerados como formas arcaicas. No presente, existem muitos estudos a respeito dos conhecimentos sobre a saúde e a doença Guaraní, cujas referências podem ser vistas, por exemplo, em Garcia $(1979,1985)$ e Buchillet $(2007)$. Porém, falta um vocabulário mais extenso e completo em todos os sentidos, para oferecer aos médicos os meios mais completos e precisos para tratar os seus pacientes. Assim seria possível desnaturalizar o conhecimento e melhorar o atendimento no serviço público, ao mesmo tempo que se pode incentivar os agentes de saúde a capacitarem-se nas línguas Guaraní, tal como alertavam Gatti e seus colegas em 1947 (p. ex.: Pícoli 2008; Pellon e Vargas 2010; Cantore 2014; Piñeiro Aguiar 2015; Falkenberg et al. 2017; Marx et al. 2020). O ideal seria reunir todos os vocabulários de 
anatomia, medicina e farmácia, publicados desde Montoya até o presente, em um único instrumento de consulta pública.

Este artigo reúne registros que contribuem para a compreensão dos "processos corporais (físico-emocionais) desdobrados em saúde-satisfação ou doença-insatisfação", tanto no passado, como na atualidade nas aldeias Guaraní Mbyá. Trata-se de concepções transmitidas entre as gerações, em "complexas negociações intersubjetivas voltadas à produção de saúde e do estado referido como 'estar alegre' (-vy'a)", como mostra muito bem Elizabeth Pisolatto (2019). Como registrou Montoya, vy'a (T:627) traduzia-se por hallarse, pegarse, detenerse, comodidad, centrado na noção de bem estar: avy'a (T:627) hállome bien, estoy acomodado. Situação muito próxima do estado de boa saúde: aguyje, aguyjei, aguyjetei (T:13) salud, sanidad, bien, bueno, bien está. Enfim, se poderia dizer: aguyjetei che rui (T:14) estoy bien acomodado, y con salud. De forma mais específica, Remorini $(2010,1060)$ mostrou critérios que os Mbyá usam para distinguir sinais e causas das enfermidades: 1) doenças leves/doenças graves; 2) doenças do corpo/doenças do espírito; 3 ) doenças de "dentro do corpo" (vísceras)/doenças externas; 4) doenças que se curam com plantas medicinais/doenças que se curam com tabaco e orações; 5) doenças próprias dos Mbyá/doenças dos jurua ("brancos").

Será apresentada uma seleção específica de vocabulários e frases Guaraní sobre "estar doente", com dois objetivos: 1) servir a história colonial da medicina e das epidemias, demografia histórica e farmacologia desenvolvidas para combater os efeitos das doenças epidêmicas, temas ainda ignorados ou pouco investigados no Brasil (Noelli 1998a, 1998b, 1999, 2000a, 2000b; Noelli e Soares 1997); 2) servir como fonte para a linguística histórica-comparativa sobre saúde-doença entre os povos falantes das línguas Tupí (Rodrigues e Cabral 2012). Poderia ser mais extenso, mas para não aumentar o tamanho do texto resisti à tentação de tratar de anatomia, lesões e da etimologia de cada expressão, não as traduzindo com mais detalhes e exemplos. Portanto, fica a sugestão para que se navegue mais pela vastidão das 1062 páginas dos dicionários de Montoya, preferencialmente usando as versões transliteradas de 2002 e 2011, mais acessíveis para quem usa algum dos três dialetos Guaraní no presente (mas sem deixar de consultar as edições anteriores, todas on-line, preferencialmente a primeira).

Este artigo está alinhado entre aqueles que defendem a história das doenças e do impacto das epidemias nas Américas sem o sentido moralizante da "lenda negra", da "visão dos vencidos" e do debate da demografia histórica entre altos e baixos números de vivos e mortos a partir de 1492 . O objetivo é compreender, em nível local e regional, o que passou com tanta gente que sofreu ou sucumbiu aos patógenos infecto-contagiosos. Não se defende mais a ideia de "solo virgem" (Crosby 1976), nem do encontro de duas entidades homogêneas, a eurasiana/ africana com imunidade e a dos povos originários sem imunidade (Jones 
2003). Defende-se aqui a necessidade de reconhecer casos vividos, como são os detalhes apresentados por Montoya e outros registros coloniais escritos que esperam ser investigados, que não citaremos aqui. Assim como foram afetados por diversas epidemias no passado, nos dias atuais os povos originários são duramente impactados pela COVID-19 neste ano de 2020, sendo evidente, mais que nunca, a necessidade de aperfeiçoar os sistemas de saúde e a formação de equipes para atuar com competência na intermedicalidade.

\section{$O$ conteúdo deste artigo}

Montoya compilou uma série de sinais, sintomas e patologias, alguns muito específicos e outros que podem ser comuns a diversas doenças, cuja análise e comparações históricas não faremos devido ao nosso objetivo de apenas apresentar os registros.

Aqui cabe comentar que muitas traduções de Montoya não foram literais, em meio à literalidade predominante. Muitas traduções foram interpretações das ideias e falas Guaraní, outras foram adaptações do discurso colonial buscando o convencimento e incutir ideias, conteúdos, regras e noções do modo de vida europeu. Outras visavam a perspectiva da luta contra o ñande reko, o "modo de ser" Guaraní, transmitindo para os missionários aprendizes da língua palavras e expressões estratégicas para a dominação colonial. No caso da saúde, temos o exemplo da luta ideológica pelo domínio colonial realizada até no campo da semântica contra o paje e a kuña paje, nomes que se poderia traduzir como médicos ou sábios conhecedores da natureza e da alma humana, mas que foram impropriamente vertidos como feiticeiro e feiticeira. Eles foram lideranças intelectuais que contestaram e criticaram abertamente o cristianismo e os missionários, denunciando-os por tentarem mudar o ñande reko.

O artigo não esgota o que está disponível nos dicionários. E a organização do texto é temática, dividida em duas partes, a serem publicadas em dois artigos separados na Revista Brasileira de Linguística Antropológica: 1) noção de saúde e doença; 2) doenças epidêmicas. Não segui uma estrutura orientada pelos sistemas de organização didática para conteúdos da Medicina. Contudo, evidentemente, procurei organizar o conteúdo em blocos temáticos mais ou menos coerentes, tentando ordenar os registros selecionados durante minhas várias leituras dos dicionários desde 1988 (cf. Noelli, Brochado e Corrêa 2018). $\mathrm{O}$ objetivo foi reunir aquilo que pareceu mais significativo ao tema proposto.

Finalmente, antes de começar, gostaria de assinalar que Montoya, de modo geral, pautou suas definições no Tesoro de la lengua Castellana, de Sebastián Covarrubias Orozco (1611). Eventualmente, citaremos as definições deste dicionário para explicar o que Montoya está expressando (virá referido como TC:352, Tesoro de la lengua Castellana, 1611, página 352). As referências das citações de Montoya também virão abrevidas, para economia de espaço (T:1, 
V:1, Tesoro de la lengua Guarani, 2011, página 1; Vocabulario de la lengua Guarani, 2002, página 1), e todas foram conferidas de acordo com as edições de 1639 e 1640. A influência de Covarrubias Orozco vai além do texto, como se percebe pelas datas e local de publicação, sendo ambos os dicionários publicados em Madri, com 28 anos de diferença, na mesma editora, a prestigiada casa Sanchez.

As palavras em Guaraní vêm destacadas com negrito, enquanto as traduções de Montoya se ressaltam com itálico: che ru raguyjei (T:217) vengo con salud. As palavras e frases de Covarrubia Orozco vêm grifadas com itálico.

\section{Doente}

A definição mais inclusiva para "doente" é hasyhasy, hasy atã, hasy para, hasy'ĩ (V:158) dolente. Aquele que está doente, cujo corpo "contém" uma enfermidade é o tasyvo (T:531, 622) enfermo (el que contiene enfermedad) (vo T:622 marca, 'continens' [cosa que contiene] ou muy enfermo, conforme a evolução da doença. Também pode ser referido como hasýva'e (T:531) el enfermo (va'e T:617 el que...). Em TC:324v temos dolor definido como um sentimiento que se hace de todo lo que nos dá desplacer y desgusto. Então poderia dizer: 1) nache reko avẽ ruguãi areko (T:104) no estoy como solía (el enfermo estar sano, o el que siempre estaba sano caer enfermo) 2) che asose mba'e rasy (T:91) es sobre mis fuerzas, mi enfermedad o dolor; 3) che rasy che mbopyryvi (T:477) la enfermedad me tiene descaecido; 4) che pohanõ (T:434) me curo; 5) añemopohanõ (T:434) curarse a sí mismo; 6) che rasy jevy (T:531) recaer.

\section{Doença e dor}

Conforme TC:352, quando a pessoa está enferma, no está en salud; quando está enfermiça, trae la salud muy quebrada, y cae muchas veces en la cama, que por otro nombre se llama achaquiento. Antes de tratar da definição de doença, veremos a definição de aguyjei (T:13) sanidad: 1) teko aguyjei (T:14, V:358) buena vida, salud; 2) aguyjetei (T:14) bueno, bien; 3) che aguyjetei (T:14) estoy bueno; 4) che ru raguyjei ( $\mathrm{T}: 217)$ vengo con salud; 5) che aguyjei guitekóvo (T:13) ando con salud; 6) aguyjetei niko amã tiri ndo'ári nde ri ra'e! (T:15) jventura ha sido llegar con salud! Um sintoma de doença poderia começar ser manifestado assim: 1) ndache aguyjetei (T:17) no estoy bueno; 2) che aguyjei jei (T:13) así así ando de salud; 3) aguyjei pokã (T:14) estoy medio bueno de salud, (jéi T:207 "se usa siempre con negación; corresponde a 'no voy, [que si], yo fuera; no me levanto, que si yo me levantara).

Os conceitos mais básicos para doença e dor são sinônimos em Guaraní, com a forma fonológica do tema asy variando conforme o contexto sintático em que ocorre: 1) asy, hasy, rasy, tasy (T:92, 150, 531, V:157, V:158) enfermedad, 
dolencia, dolor, significando também: pena, dificuldad, trabajo, vehemencia, em circunstâncias onde o sintoma é persistente e faz sofrer; 2) marã, mara'a, mbara'a, mba'e asy, mba'e rasy (T:296, 297, 325, V:171) enfermedad, dolencia (mba'e T:325 cosa, algo, se añade a los substantivos); 3) tasy porokutu (T:531, V:158) dolor agudo. Os primeiros sintomas de doença eram sentidos, incluindo uma localidade específica: 1) ovu che mba'e rasy (T:624) brota mi enfermedad;2) kieve che rasy (T:250) desde aqui estuve enfermo; aqui empecé a enfermarme (ki, kie T:250 aqui); 3) eupeve che rasy (T:122) desde ahi estuve enfermo. E quando todo o corpo ficava afetado diriam: 1) che rete opa katu che rasy ohu (T:175) hame cogido todo el cuerpo la enfermedad; 2) mba'e rasy che hu (T:175) todo el cuerpo me ha cogido la enfermedad; 3) tasy che pysypa (T:479) hame cogido toda la enfermedad. Poderia ser apenas um resfriado: ro'y che pysy, che ro'y a, che karasy (V:350) resfriarse (que também se pode traduzir como: o frio me pegou, tenho dor de cabeça).

Temos exemplos relativos a cansaço e indisposição relativos a doenças, citando apenas dez exemplos: 1) perder apetite: nache mbojuru a'éi che rasy (T:220) la enfermedad me há quitado el sabor, o apetito; 2) ter sono e sonolência: topehýi (T:584, V:279) sueño, che ropehýi (T:584) tengo sueño, che rope piratãi (T:584) estoy soñoliento; 3 ) ficar cansado: che kane'õ T:236, V:88 estoy cansado, cansarse che ose che kane'õ T:236 estoy muy cansado (kane'õ T:236, V:87 cansacio); 4) mialgia: tasyrõ mba (T:531) molimiento de todo el cuerpo, che rasyrõ mba (T:531, V:157, 280) estoy molido todo, doler todo el cuerpo; 5) sensação de aperto, esmagamento do corpo: apururũ che rasýramo (T:454) crújeme el cuerpo con la enfermedad; 6) perda de energia: nache piratãvéi ( $\mathrm{T}: 411)$ estoy descaecido, che piratãmba, che pitãpávi (T:411) ya se me han acabado mis fuerzas; 7) fraqueza: che mokangy che rasy (T:237) hame debilitado la enfermedad; 8) fraqueza: che kãngo kãngo che rasy (T:237) estoy quebrantado con la enfermedad; 9) tristeza e depressão: ague rai guitékovo (T:131) estoy triste, marchito, enfermo, o melancólico; 10) tumeficar: che rasy nache mbojaity potári (T:199) tiéneme entumecido la enfermedad. Além disso, Montoya registrou inúmeros casos de sintomas, sinais, fraturas, cortes e diversas lesões que não serão citados aqui.

Foram registrados inclusive alguns exemplos de expressões e respostas de doentes: 1) ái, aju, atã (T:15, 22, 93) interjección dolentis [del que sufre], heguã ãngai! (T:160) interjección de mujer 'dolentis' [que se duele]; 2) áivetei (T:18) malamente; 3 ) aivĩ (T:18) consumido. E, também, de doentes ou pessoas no contexto da doença: tasy'e' $\mathbf{y}$ mbire tamo ereju (T:123) oh, si hubieras venido antes de la enfermedad.

\section{Casos individuais}


Para os casos individuais: 1) che marã, che rasy (T:296, 531) estoy enfermo; 2) hasýpe che rui ( $\mathrm{T}: 532)$ estoy enfermo, y con pena; 3) che marã guitekóvo (T:296) ando enfermo, consumido; 4) che rasyse (T:531) soy enfermizo; 5) che rasyrasy'ĩ, che reko aguyjeie' ỹ tapia, che rasy herãherãnĩ, che rasyse, che aruru (V:171) enfermizo andar; 5) che marãnĩ guitekóvo (T:300) ando enfermizo; 6) che retépe areko mba'e asy (T:574) tengo enfermedad en mi сиегро (tete T:575 сuеrpo).

O enfermiço poderia eventualmente ser crônico e ter uma tasy tapia (T:531) enfermedad continua. Com a persistência dos sintomas, diria che rasy nunga, che rasy vevúi, che rasy atã, che rasy pyry, che rasy herã ñõte (T:354, 531, $\mathrm{V}: 158)$ estoy medio enfermo, doliente un poco, dolor lento. Poderia exclamar, se ficasse sem mobilidade: 1) che rasy omorãgue che ho (T:491) la enfermedad me estorbó mi ida; 2) guasy guenoĩramo ndoúri (T:183) por estar enfermo no vino.

A pessoa não estaria fingindo: nache rasy rui ruguãi (T:504) no es de burla mi enfermedad, añemboasyasy a'u (V:217) hacerse enfermo, fingiendo.

Com a evolução diria: 1) tasy ojeroviári (T:501) prevalece la enfermedad; 2) oñeapyrũ ngõte che rasy (T:74) hase reforzado mi enfermedad; 3) amboa'e voi che reko asy herekóvo (T:142) hago que persevere mi enfermedad; 4) amboa'e voi che reko asy herekóvo (T142) hago que persevere mi enfermedad, o trabajo; 5) oñemboapyrytarũ che rasy (T:77) auméntase mi enfermedad; 6) ymaguarérava'e (T:638) mucho ha que estoy enfermo.

E, com o agravamento, os exemplos aumentam: 1) che rasy, che rasy été, che rasy aiai, aivete'i che rasy (T:531, V:158) estoy muy enfermo, doliente mucho; 2) che hu katu mba'e asy (T:531) ando muy enfermo; 3) marã marã etei ahẽ rekóni (T:296) está muy enfermo, o ruin; 4) oñepũ ngatu oúpa (T:452) está muy enfermo; 5) tasy te'õ reruháva, poromboekyise, porojuka, poromoe' $\tilde{\text { o, }}$ che mbojekohu te'õ rehe che rasy (V:171) enfermedad mortal; 6) che rerokaka te'õ upe che rasy (T:233) voy acercándome a la muerte [por enfermedad]; 7) haĩme che re' õ (T:562) por poco me muero; 8) te'õ rovapy'ĩme aiko (T:562) estoy a pique de morir; 9) koĩrĩ che re'õ viñã (T:255) estuve a pique de morir; 10) koĩkoĩ amano viñã (T:255) muy cerquita estuve de morir; 11) koĩ koĩ namanói, haĩme che re'õ $(\mathrm{T}: 255,562)$ por poco me mиero (koĩ T:255 muy cerca, lo mismo que koĩ eteĩ, te'õ T:563 desmayo).

Se houvesse melhora, se diria: 1) oku'e ku'e che rasy (T:271) remitese la enfermedad; 2) ijapyryve che rasy (T:78) aplácaseme la enfermedad, o dolor; 3) ijaryve che rasy (T:89) háseme aplacado la enfermedad.

Finalmente, sem melhora do quadro, a pessoa poderia: 1) amano ai (T:293) morir mal; 2) nache porãgi (T:434) no me curan, no tengo cura; 3) mba'e ipohãngỹmbae (T:434) cosa incurable, sin remédio; 4) amano ei (T:293) morirse sin enfermedad, sem doença pré-existente ou por causa indeterminada. 
E, antes de falecer, poderia dizer: che rupiára areko che retépe (T:505) la causa de mi enfermedad tengo dentro de mi ou che rasy renoĩna (T:183) teniendo yo mi enfermedad.

Montoya mostrou um ambiente onde a magia do catolicismo estaria presente: ehovasa che ra'y (T:587) dicen cuando piden un evangelio al enfermo. $\mathrm{O}$ contrário, tereñemombe'u nde rasy ypýramo ha'e jepe ichupe ha'e aroirẽ imanóni (T:87) dijele que se confesase luego que enfermó y no quiso, y así se ha muerto sin confesión. Poderia receber os óleos da extrema-unção: amongy ñandy rovasapy hasýva'e rehe (T:318) poner olios al enfermo (ñandy T:361, 530, V:11 aceite de frutas, y árboles; mongy T:318 ungir, poner; unción; nandy mongy, pitu T:421, V:392 unción). Os missionários explicavam a presença das doenças com perspectivas diferentes em relação às Guaraní de saúde/doença: 1) ñande renopu'ã Tupã ñande mboasýramo (T:165) amedróntanos Dios con enfermidades, que pode ser traduzida de outra forma: Deus nos sara, levanta/ avisa (ao invés de ameaça) quando estamos doentes; 2) akoi nde rasý haguéra Tupã nde renopu'ãháva ñõte (T:165) la enfermedad que tuviste no fué más que amago [gesto, ameaça] de Dios.

\section{Epidemias}

Aqui começo a considerar as doenças agudas, infectocontagiosas e coletivas, que se espalham velozmente através de contágio direto e indireto até contagiar grande número de pessoas, para depois de determinado tempo se extinguir. Montoya expressa claramente a noção de contágio dentro da teoria do "ar mal ou corrompido" que circulava na Europa desde os Gregos (Grmek, 1989), referindo-se a peste e contágio de acordo com os termos de CovarrubiasOrozco (TC:234v): 1) contagión, la enfermedad pegadiza; 2) contagioso, el que tiene mal que se pega; 3) pegajoso, el que se pega; 4) corromperse uno, es desmayar, yendose de cámaras. Na primeira metade do século XVII, a definição de "peste" era: enfermedad contagiosa, que comunmente se engendra del aire corrompido, del latín, pestis. Pestilencia, lo mesmo que peste. Pestilencial, lo que puede causar peste. Pestilente y pestifero. Apestado, lo que está tocado de la peste. Apestarse un lugar, tener peste (TC:587). Aqui surge a primeira dúvida, se Montoya usa apenas o sentido genérico de doença infectocontagiosa, ou se também se referiu à peste bubônica, como será mostrado na segunda parte deste artigo, quando se tratará das doenças específicas. Esta dubiedade acompanha a definição de outras doenças.

O contágio viria com os ventos: yvytu oipeju tasy (T:655) el viento lleva las enfermidades (yvytu T:655 viento, peju T:404 soplar). A noção de lugar contagiado aparece com yvy asy, mañondody ko yvy (V:171) enferma tierra. O lugar ainda não contagiado também aparece: tasy kyvõ ndoúri (T:288) no ha venido acá la enfermedad, nimarãni che róga (T:296) está mi casa entera; no 
hay enfermedad, o cosa mala, en mi casa. A duração da enfermidade também era considerada: ára teko asy rerekuára (T:5) tiempo enfermo, y calamitoso, has yete rako ang (T:532) tiempo trabajoso.

Montoya criou expressões sobre a propagação de "mal contagioso", de "doença que se pega": 1) mba'e asy jepota, mba'e asy jepota va'e, mba'e asy oja va'e, mba'e asy oja ojaréva'e, mba'e asy ojepotáva'e (T:531, V:111) mal contagioso; 2) Mba'e ñemboja (T:193) cosa que se pega, enfermedad; 3) mba'e asy ojáva'e (V:312) pegajosa enfermedad; 4) mba'e asy oiko, tasy ja reko, tasyvo ei, tasy ohu katu, mba'e tasy okua (V:115) correr enfermedad (que poderiam ser traduzidas como "doença que anda, temos doença por toda a parte, enfermidade que se pega bem fácil"). Também expressões individuais, da pessoa se considerando contagiada: 1) mba'e asy ojepotáva'e aiporara, mba'e asy jepotare che hu (T:531, V:111) padezco mal contagioso; 2) omboja che ri guemimborara (T:193) pegóme su enfermedad; 3 ) che reko asy oñemboja'a chéve (T:6) aumentase mi enfermedad.

Na língua Guaraní, peste e mortandade são sinônimos, sempre com o tema pab, pa, mba (T:391, V:197) fin, acabamiento, muerte: 1) mbáva, tasy ai (V:319) peste; 2) mbáva, páva (T:329, 391 V:281) mortandad, acabamiento. A definições também informam a magnitude dos seus efeitos: 1) mbáva niko (V:212) haber pestilencia; 2) mbavai oiko (V:319) peste haber; 3) mba'e manẽma (V:319) pestilente, cosa mala (manẽ, panẽ T:293, 394, V:145 desdicha, desventura); 4) mba vetei kuái (T:329, 391 V:281) gran mortandad hay.

Sobre o espalhamento da epidemia temos algumas traduções: 1) oñeirumõ che rasy, okakuaa (V:119) crecer la enfermedad; 2) mba'e asy oky rusu ore rehe (T:283) llueve enfermedad sobre nosotros (chove "grande" = muito); 3) mbapára amyrĩ rogué ipichyvy ( $\mathrm{T}: 415)$ ha quedado la casa del difunto espantosa; 4) ndipo vyvy retéi mbya okuápa tasy'a ri (T:627) todos están caídos de enfermedad; 5) ipichyvy táva'a oúpa hasypávamo (T:415) está el pueblo espantoso con tantos enfermos.

A situação era difícil, pois com o acometimento generalizado havia falta de recursos e gente saudável para cuidar dos doentes: 1) tasy che reity (T:190) la enfermedad me ha derribado; 2) ndache reropu'ãi che rasy (T:452) no me levanto ya de la enfermedad; 3 ) che rerovyhave'ỹme (T:627) no hay quien me levante, o me ayude en enfermedad; 4) naku'éi guitúpa (T:271) no me meneo (dice el enfermo); 5) che ku'e ndoipotári che rasy (T:271) no me deja menear la enfermedad; 6) che mỹi ndóipotári che rasy (T:271) no me da lugar la enfermedad a que me menee; 7) ambojaupi hasýva'e (T:204) hacer levantar la cabeza al enfermo; 8) tasyvo ijojog yma (T:216) ya tiene hipo el enfermo (jojog T:216, V:226 hipo); 9) ndijaupirivéi hasýva'e (T:204) ya no levanta cabeza al enfermo; 10) ndipovy tasývo (T:422) no se levanta al enfermo; 11) 
nache moñaropu’ãvéi che rasy (T:363) ya no me levanto más. A alimentação e a hidratação ou a sua falta eram críticas: 1) y'uhéi aiporara (V:303) padecer sed; 2) ndache rembi'u hói guikyhyjávo (T:172) la enfermedad me ha quitado el comer; 3) nache mboaguyjéi (T:13) el no comer es causa que yo no sane; 4) che pĩ che rasy (T:413) hame dejado en los huesos la enfermedad; 5) apaguy, oñembohetyma guyguy (T:56, 134) bambalearse el flaco, o enfermo; 6) aguyvi (T:13) caerse de flaco; 7) che rako pevei (T:524) estoy trasijado [muy flaco]; 8) che kãnguerĩ (T:237) estoy muy flaco; 9) kangy (T:237) débil, flaco; de poca fuerza.

Durante a epidemia a situação poderia ficar muito dura: 1) ore mboapakúi tasy (T:57) la enfermedad nos ha derribado; 2) amano pyrĩ (T:475) estoy para morir; 3) mbapára róga irui katu (T:504) está la casa del difunto en silencio; 4) táva irui katu tasýva ri (T:503) está pueblo en silencio por los enfermos que hay.

Depois da epidemia mortífera, o terrível saldo entre os sobreviventes: 1) ore monungarĩ tasy (T:354) la enfermedad nos ha apocado, consumido; 2) nache moamonguéri tasy ( $\mathrm{T}: 35)$ la enfermedad me ha llevado mi gente; 3) ndache retavéi (T:168) hace consumido mi gente; 4) che mbotyavo mba'e rasy (T:604-605) hame acabado mi gente en la enfermedad; 5) che johuamoguára ojejarog (T:175) hanse acabado los de mi parcialidad (ajarog, ojejarog, jarog V:275 mermar, merma, menoscabar, menoscabo); 6) añemomba (T:391) ya se han acabado los míos, mi gente; 7) opa ipavi (T:391) todo se acabó; todos se han muerto; 7) koĩ yvága rúri chéve (V:347) rematado estar de enfermedad. Assim como está evoluindo a segunda onda da COVID-19 em outubro de 2020, há evidência de recorrência no Guairá: che rero'a jevy; che rasy oñeirumõ (V:119) crecer la enfermedad recayendo.

O sobrevivente testemunhou o fim da epidemia: mbáva opig, ijapyryve mbáva, nahe'õvéi (V:46) aplacar mortandade. E poderia reclamar: 1) onduru nduruhápe mba'e asy che hu (T:348) tropel de trabajos, o enfermedades, me han venido; 2) nache poakári che rasy rereko (T:422) ya no puedo sufrir la enfermedad.

\section{Cuidado}

O registro de várias expressões mostra a importância do cuidado dos doentes: 1) aĩ hasýva'e pýri (T:183) acompaño al enfermo; 2) aĩ hasýva'e irũnamo, aiko ipýri, añemoirũ hasýva'e rehe (V:13) acompañar al enfermo (irũ T:188 compañero). E, poderia se perguntar como ia a saúde: aguyjepeípe ereiko? (T:13) ¿estás con salud?

Também de exclamações sobre o que passou com os enfermos: 1) che pĩ ngatu hasykuéra (T:413) mucho he sentido su enfermedad; 2) añemomboriahu nde rasy recháka (T:444) aflijóme de verte enfermo; 3) ti’ã ahẽ guasýramo 
pa! (T:580) ¡que será del enfermo!; 4) atáipa ahẽ rasýramo ra'e ra! (T:93) ¡oh, cómo me pesa que esté fulano enfermo!

Há registros sobre a alimentação e limpeza de doentes: 1) hasýva'e mba'e aparyku ho'u katu (T:59) los enfermos comen bien cosas líquidas; 2) nache mboaguyjéi (T:13) el no comer es causa que yo no sane (aparyku T:59 desleir); 2) haĩmbetei nde re'õ ha'e, koĩ te'õ águi nde reko guijávo, te'õ pópe ereiko ha'e, pyrĩ te'õ ipokohave'ỹ nde rehe guijávo, ha'imbete'i nde r'õ a'e, koĩ te'õ agui nde guijávo, nderekovéi séne, eremanõne (T:563, V:141) desahuciar el enfermo (quando há a palabra te'õ T:563 desmayo, também significa inconsciente). E, também, tocar ou movimentar ou não o paciente: añatõi (V:274) menear (ñatõi T:363 tocar), che mbokosog, che mbokotog (T:258) me menean, menéanme; namboapagúyi (T:56) no le meneo (kosog, kotog, kotõ T:258 vaivén, meneo, menear). A hidratação era um cuidado essencial: amboy'u (T:633) darle a beber; ay'u (T:633) beber; y'u (T:633) bebida de água; y'héi (T:633) sed; che y'uhéi (T:633) tengo sed; che y'uhéi ai ai (T:633) tengo mucha sed.

\section{Convalescência/Cura}

A evolução para o estado de saúde possui várias expressões, começando por: 1) ndakuerávi (T:272) no estoy sano; 2) nda'ei guikuerávo rangẽ (T:272) aún no estoy sano. Depois, a melhoria: 1) akuera vatã (T:272) estoy medio sano; 2) asãndog sãndog che rasy (T:507) remite algo mi enfermedad; 3 ) tasy ivevúi ko'yte (T:621) ella pasa la enfermedad; 4) mba'e porarahára ivevúi (T:621) están los enfermos aliviados; 5) tasy ivevúi ko'y te (T:621) ya pasa la enfermedad.

A convalescência e a cura: 1) kuera (T:272) convalecencia; 2) che kuera (T:272) mi convalecencia; 3) ipovy ramo tasývo (T:422) ya anda convaleciente; 4) akuera kuera (T:272) estoy mejor; 5) añaropu'ã ramo (T:362) me empiezo a levantar, estoy convaleciente. A melhoria é encontrada em otras falas: 1) ñaropu'ã, ñarapu'ã (T:362) levantarse el enfermo, o sano, de la cama, sentarse en la cama; 2 ) akuera vamo (T:272) ahora salgo de la enfermedad; 3 ) akuera (T:272) estoy sano; 4) amonguera (T:272) sanarlo; 5) nimarãni che rete, nache marãni (T:296, 297, V:360) estoy sano. Com a cura, diria: aiko ete ko'yte che rasýgui (T:120) estoy ya recio de mi enfermedad (ete T:120 bueno, recio).

A busca pela cura também se revela na linguagem relativa às drogas medicinais, cujo nome genérico se traduz por pohãng, mohãng, mohãngámo herekopy (T:434, V:272) medicina, remédio. A cura usando uma droga é pohanõ (T:434) curar e, como a maioria dos remédios são líquidos, o seu nome genérico é pohãngy'u ou u (T:434, 612, V:70) bebedizo. A sua ingestão era añemopohãngy (T:612) tomé bebedizo, e quem aplicava diria: 
amopohãngy'u (T:612) dile bebedizo. A palavra bebedizo já é má tradução, por ser desqualificadora, desqualificadora, pois o termo na época era um equivalente de "poção" que as mulheres faziam para seus maridos ficarem com elas ou para não saírem com outras pessoas, induzidas por viejas hechiceras e, ainda, poderia ser um veneno (TC:135v).

A pessoa com o conhecimento para produzir drogas medicinais era o pohanõngára (T:434) médico (a tradução literal é "fazedor de remédios"). Se fosse bom, era considerado um porapohanõhára ekatu, porapohanõhára avye'̃̃, porapohanõngára (V:12, 272) acertado médico. Como o contexto da doença infecto-contagiosa exigiria prédicas, cantos, danças, uso de fumaça de tabaco soprado no doente, massagens e outras práticas médicas, sendo essa pessoa conhecida como paje ou kunã mbaje (T:394) hechicero ou hechicera duas traduções carregadas de preconceito e estratégias para eliminar a sua liderança intelectual e política.

A pessoa que não se curava da sua doença poderia dizer: 1) nache pohãngi (T:434) no me curan, no tengo cura, o remedio; 2) mba'e ipohãngỹmbae (T:434) cosa incurable, sin remedio; 3 ) mba'e ipohãmbyre' ̃̃ ko (T:434) esto es cosa a que no se aplicado medicina. Neste caso, surge a ação de quem faz e/ ou aplica a droga: 1) aipohanõ (T:434) yo le curo; 2) che pohãno (T:434) me curo; 3) añemopohanõ (T:434) curarse a sí mismo; 4) amopohanõ (T:434) hacer que le cure.

\section{Considerações finais}

É possível afirmar que a linguagem da saúde e da doença Guaraní é muito mais ampla que o registro de Montoya. O conteúdo reunido neste artigo é valioso pelas várias razões apontadas ao longo do texto, tanto na excelência linguística, quanto na obra de alguém paradoxalmente dividido entre o dever do servidor colonialista e o afeto inúmeras vezes demostrando pelas pessoas que pretendia transformar e incorporar aos súditos do rei espanhol.

Interessa-me particularmente reunir informações para a história intelectual dos Guaraní, da capacidade de guardar e transmitir conhecimentos entre as gerações na longa duração. A arqueologia já mostrou que a presença deles na Bacia Platina alcança até 2 mil anos antes do presente (Bonomo et al. 2015). E, de uma perspectiva comparada dos registros arqueológicos e históricos, fica evidente que eles também conservavam estruturas de conhecimentos até mais antigas que eles próprios, como se pode verificar através das reconstruções da linguística histórico-comparativa, de onde se pode perceber os conteúdos e práticas dos Guaraní em relação com outros povos falantes das línguas do Tronco Tupí.

Desde a década de 1990 (Noelli 1993, 1998a), me interessa compreender como os Guaraní usaram seus conhecimentos médicos e farmacêuticos para 
enfrentar as doenças infecto-contagiosas a partir do ano 1500, talvez antes. A comparação de informações históricas e a regularidade dos registros linguísticos de tempos e lugares diferentes permitem levantar a hipótese de que a sistemática da área médica era algo intrínseco do ñande reko, domínio dos paje e das kuña paje e das(os) mba'e kuaaparete (V:356) mucho sábio (kuaa $\mathrm{T}: 264$ conocer, saber, entender, comprender, certidumbre). Com o passar dos anos e do acúmulo de conhecimentos, ficou evidente que o estabelecimento em novos locais era precedido por levantamento detalhado dos recursos naturais, incluindo o inventário das plantas medicinais.

O desafio é compreender como funcionava essa sistemática e como eram as comunidades de prática que acumulavam e transmitiam tais conhecimentos. Mas também é importante entender quais os efeitos da colonização que levaram à desestruturação e desagregação das constelações de assentamentos Guaraní, das epidemias que mataram inúmeros sábios e, mais tarde, forçaram a constante mudança de assentamento devido às pressões e conflitos territoriais e fundiários, que se acirraram especialmente a partir do século XIX. Quais as consequências disso na conservação da linguagem e do conhecimento médico? Não se trata de uma simples questão de perda, mas de como aqueles que sobreviveram, talvez em muitos lugares sem nenhum especialista plenamente formado ou, eventualmente, com pessoas com conhecimentos parciais, conseguiram lidar com as doenças? E como a mudança para ecossistemas povoados com plantas diferentes das conhecidas afetou a produção das drogas medicinais?

Enfim, tanto no passado como agora, a maioria dos lugares precisa dos conhecimentos médicos e farmacêuticos Guaraní, mais a capacidade ambiental para oferecer as plantas para exercer as práticas milenares. Ao mesmo tempo, também é necessária a relação com os sistemas públicos de saúde no Brasil, Argentina, Uruguai e Bolívia, para resolver os casos que não se resolvem no teko'a, com equipes capacitadas nos dialetos para ouvir os doentes e compreender suas necessidades físicas e da alma.

\section{Agradecimentos}

Ao $\mathrm{CNPq}$ pela bolsa de doutoramento que subvencionou a pesquisa que originou este artigo (1998\2001). A sua primeira versão é de 1998, como parte da minha tese interrompida por causas alheias à minha vontade, orientada por John Manuel Monteiro na UNICAMP, com quem debati intensamente o impacto das epidemias sobre as populações Guarani e Jê no sul do Brasil, no período colonial. John lamentava a falta de estudos neste campo de conhecimento tão importante, mas era otimista quanto ao seu desenvolvimento futuro. Bartomeu Melià me incentivou a fazer este projeto em 1994, sugerindo que os registros de Montoya eram itens obrigatórios. Graciela Chamorro leu em 1998 a minha primeira organização desses registros e fez algumas traduções alternativas, 
usadas neste artigo. Agradeço à Beatriz do Santos Landa e André Luís Ramos Soares, por abraçarem comigo e com dedicação a difícil tarefa de ordenar tematicamente o meu levantamento nos dicionários de Montoya e Carlos Gatti, entre 1990 e 1992. À Ana Suelly Cabral pelo incentivo para terminar o texto. Ao médico Eduardo Siqueira, Professor da UMass-Boston, e aos pareceristas da RBLA, pelas correções e sugestões. À Marianne Sallum pelas ideias nessa versão final, durante o confinamento da segunda onda da COVID-19 em Lisboa, em outubro de 2020.

\section{Referências}

Buchillet, Dominique. 2007. Bibliografia crítica da saúde indígena no Brasil (18442006). Quito: Ediciones Abya-Yala.

Bonomo, M.; Angrizani, R. C.; Apolinaire, E.; Noelli, F. S. 2015. A model for the Guaraní expansion in the La Plata Basin and littoral zone of southern Brazil. Quaternary International http://doi.org/10.1016/j.quaint.2014.10.050

Cantore, Alfonsina. Antropología y la desnaturalización del sistema de salud: sobre la concepción de enfermedad de comunidades Mbyá en Misiones. En Letra 1:142-163.

Covarrubías-Orozco, Sebastián. 1611. Tesoro de la lengua Castellha, o Española. Madrid: Luis Sanchez.

Falkenberg, Mirian B.; Shimizu, Helena E.; Bermudez, Ximena P. D. 2017. Representaciones sociales de la atención sanitaria de la población indígena MbyáGuaraní por parte de trabajadores de la salud. Revista Latino-Americana de Enfermagem https://doi.org/10.1590/1518-8345.1505.2846

Garcia, Wilson G. 1979. O domínio das plantas medicinais entre os Kayová de Amambai: problemática das relações entre nomenclatura e classificação. Dissertação de Mestrado, Universidade de São Paulo.

Garcia, Wilson G. 1985. Introdução ao Universo Botânico dos Kayovás de Amambai: Descrição e Análise de um Sistema Classificatório. Tese de Doutorado, Universidade de São Paulo.

Gatti, Carlos. 1985. Enciclopédia Guarani-Castellano de Ciencias Naturales y Conocimientos Paraguayos. Asunción: Arte Nuevo Editores.

Gatti, Carlos; Rojas, Teodo, Bertoni, A. de W. 1947. Vocabulario Guaraní-Español para uso médico. Asunción: Edición de los autores.

Grmek, Mirko D. 1989. Diseases in the Ancient Greek World. Baltimore: Johns Hopkins University.

Jarque, Francisco. 1900. Ruiz de Montoya en Indias (1608-1652). Madrid: Victoriano Suárez, volume 1.

Marx, Javier; Acosta, Lucrecia; Deschutter, Enrique J.; Bornay-Llinares, Fernando J.; Sotillo-Soler, Víctor; \& Ramos-Rincón, José M. 2020. Syphilis and HIV infection in indigenous Mbya Guarani communities of Puerto Iguazu (Argentina): diagnosis, contact tracking, and follow-up. Revista do Instituto de Medicina Tropical de São 
Paulo https://doi.org/10.1590/s1678-9946202062019

Melià, Bartomeu. 2011. Montoya saca a luz su Tesoro de la lengua Guaraní. In: Tesoro de la lengua Guaraní. ix-xlv. Asunción: CEPAG.

Montoya, Antonio R. 1639. Tesoro de la lengua Guaraní. Madrid: Juan Sanchez.

Montoya, Antonio R. 1640. Vocabulario de la lengua Guaraní. Madrid: Juan Sanchez.

Montoya, Antonio R. 2002. Vocabulario de la lengua Guaraní. Asunción: CEPAG.

Montoya, Antonio R. 2011. Tesoro de la lengua Guaraní. Asunción: CEPAG.

Noelli, Francisco S. 1998a. Múltiplos usos de espécies vegetais pela farmacologia Guaraní através de informações históricas. Diálogos, Revista de História http:// periodicos.uem.br/ojs/index.php/Dialogos/article/view/37489

Noelli, Francisco S. 1998b. Disease and demography in the Americas, por John W. Verano \& Douglas H. Ubelacker (Eds.). Revista de Arqueologia 11:144-146.

Noelli, Francisco S. 1999. Born to die. Disease and New World conquest (1492-1650), por Noble D. Cook. Revista do Museu de Arqueologia e Etnologia https://doi. org/10.11606/issn.2448-1750.revmae.1999.109355

Noelli, Francisco S. 2000a. Secret judgements of God. Old World diseases in Colonial Spanish America, por Noble D. Cook \& W. George Lovell (Eds.). Revista de Antropologia https://doi.org/10.1590/S0034-77012000000100016

Noelli, Francisco S. 2000b. Comentário d' "A população nativa da América do Sul”. Revista do Museu de Arqueologia e Etnologia https://doi.org/10.11606/issn.24481750.revmae.2000.109393

Noelli, Francisco S.; SOARES, André L. R. 1997. Para uma história das epidemias entre os Guarani. Diálogos, Revista de História http://periodicos.uem.br/ojs/index. php/Dialogos/article/view/37426

Pellon, Luiz H. C.; VARGAS, Liliana A. 2010. Cultura, interculturalidade e processo saúde-doença: (des)caminhos na atenção à saúde dos Guarani Mbyá de Aracruz, Espírito Santo. Physis, Revista de Saúde Coletiva https://doi.org/10.1590/S010373312010000400017.

Pícoli, Renata P. 2008. Saúde, doença e morte de crianças: um olhar segundo a percepção dos Kaiowá e Guarani. Tese de Doutorado, Universidade de São Paulo.

Pissolato, Elizabeth P. 2019. Saúde e doença em aldeias Guarani: lidando com emoções. Intersecções https://doi.org/10.12957/irei.2019.47255

Piñeiro Aguiar, Eleder. 2015. Educación médica para la inclusión intercultural desde un estudio de caso entre los Mbyá-guaraní. Educación Médica Superior, 29(4):824831.

Remorini, Carolina, 2010. Hacer crecer un niño (Mitã ñemongakuaa): el cuidado de la salud de los niños y las transformaciones en el Mbya reko. Antíteses http://dx.doi. org/10.5433/1984-3356.2010v3n6p1047

Rodrigues, Aryon D.; Cabral, Ana S. A. C. 2012. Tupían. In: Campbell, L.; Grondona, V. (eds). The indigenous languages of South America, v. 2. Boston: Moutnoellion de Gruyter. pp. 495-574. 